\title{
Recurrent hyperkalemia in patients with chronic kidney disease and hepatitis $C$ treated with direct antiviral agents
}

\author{
Taotao Yan ${ }^{1,2+}$, Jiuping Wang ${ }^{3+}$, Juan $\mathrm{Li}^{1,2}$, Shan Fu ${ }^{1,2}, \mathrm{Yi}_{\mathrm{C}}$ Chen ${ }^{1,2}$, Chunhua Hu ${ }^{1,2}$, Rou Zhang ${ }^{1,2}$, Zhen Tian ${ }^{1,2}$,
} Fahui Zhao ${ }^{4}$, Jun Dong ${ }^{5}$, Jinfeng Liu ${ }^{1,2}$, Yuan Yang ${ }^{1,2}$, Tianyan Chen ${ }^{1,2}$, Yingren Zhao ${ }^{1,2^{*}}$ and Yingli He $\mathrm{e}^{1,2^{*}}$

\begin{abstract}
Background: Sofosbuvir is the keystone of direct antiviral agents for the chronic hepatitis C (CHC). The safety of sofosbuvir in patients with stage 4-5 chronic kidney disease (CKD) needs further observation in real world.

Case presentation: Thirty-three patients with stage 5 CKD and hepatitis C virus (HCV) infection from 2 hemodialysis centers accepted sofosbuvir based treatment as we reported previously. Serum potassium concentrations were tested every 4 weeks or on demand. Ten of 33 patients showed recurrence of hyperkalemia. We summarized the characteristics of hyperkalemia occurrence in these 10 patients. Overall, 24 episodes of hyperkalemia were observed in these 10 patients, 21 were under treatment and 3 were after treatment. Patients with or without hyperkalemia before sofosbuvir treatment didn't show significantly differences in the median frequencies of hyperkalemia episodes during the observation period (3.5 vs. 2, $p=0.264$ ).
\end{abstract}

Conclusions: Patients with stage 5 CKD and HCV infection treated with sofosbuvir based regimens, even halved sofosbuvir, should be taken caution and closely monitoring serum potassium and renal function is necessary.

Keywords: Chronic kidney disease, Sofosbuvir, Hyperkalemia

\section{Background}

Hepatitis $\mathrm{C}$ virus (HCV) infection is a leading cause of chronic liver disease, cirrhosis, and hepatocellular carcinoma. Patients with chronic kidney disease (CKD), especially those with end-stage renal disease (ESRD) on hemodialysis, present high prevalence of HCV serum positivity. Currently, since the high efficacy and well tolerance to direct antiviral agents (DAAs), antiviral treatment is recommended for all patients infected with $\mathrm{HCV}$, including those with stage 4-5 CKD on hemodialysis and waiting for renal transplantation [1].

Studies showed that sofosbuvir based regimens were highly efficacy and well tolerance in patients with $\mathrm{HCV}$ and stage 4-5 CKD $[2,3]$. However, the safety of sofosbuvir, mainly sofosbuvir-derived metabolites in patients

\footnotetext{
* Correspondence: zhaoyingren@mail.xitu.edu.cn;

heyingli2000@mail.xjtu.edu.cn

${ }^{\dagger}$ Taotao Yan and Jiuping Wang contributed equally to this work.

'Institution of Hepatology, First Affiliated Teaching Hospital, SOM, Xi'an

Jiaotong University, Xi'an City, China

Full list of author information is available at the end of the article
}

with CKD remains an opening question. In the safety pharmacology studies of sofosbuvir, high dose of GS9851 (the parent of sofosbuvir) inhibited potassium current by approximately $13 \%$ at $159 \mu \mathrm{g} / \mathrm{mL}$ [4]. Patients with CKD are more prone to imbalance of homeostasis, such as metabolic acidosis, hyperkalemia. Based on our knowledge, up to now, serum potassium in patients with CKD treated with sofosbuvir has not been reported.

Previously, we reported a nosocomial HCV outbreak in Zhen'an County Hospital, Shaanxi Province, China, in January 2016 [5]. Thirty-three patients with hepatitis C and stage 5 CKD on hemodialysis were treated with half dose of sofosbuvir $(200 \mathrm{mg})$ and full dose of daclatasvir $(60 \mathrm{mg})$. All patients completed 24-week treatment and additional 12-week follow-up. This special population provided a unique opportunity for us to observe the serum potassium concentration during sofosbuvir treatment. Serum potassium concentrations were regular tested every 4 week or on demand during the treatment course and post-treatment up to 12 weeks. The levels of 
serum potassium 3 months prior to treatment were retrieved from the medical record database of the hospitals. Hyperkalemia was defined as serum potassium concentration higher than $5.5 \mathrm{mmol} / \mathrm{L}$. Ten out of 33 were reported recurrence of hyperkalemia. We report hyperkalemia occurrences of these 10 patients with CKD and $\mathrm{HCV}$ infection who received sofosbuvir-based regimens.

\section{Case presentation}

\section{Baseline characteristics}

General information, treatment regimens, comorbidity, dialysis history, co-medication and adverse effects (AEs) were retrieved form electronic medical record. Means were used to describe quantitative variables and medians were used to describe the number of hyperkalemia episodes. All statistical analyses were performed using SPSS 16.0. Patients' demographics were presented in Table 1 . There were 7 males and the average age was $49.2 \pm 14.5$ years old. The average dialysis period was $2.85 \pm 1.17$ years. Drug-drug interaction was searched in HEP Drug Interactions database reserved by the University of Liverpool (data from the website: http://www.hep-druginteractions.org). The medicines combined with DAAs in these 10 patients do not present clinically significant interaction with sofosbuvir. Seven patients took DAAs in combination with at least one of the following medicines, nifedipine, carvedilol and amlodipine, which is reported potential interaction with daclatasvir. Carvedilol is contraindicated in patients with severe liver impairment. All of the patients were mild liver damage and the liver function returned normal during the antiviral treatment.

\section{Occurrence of hyperkalemia}

Figure 1 showed the detailed hyperkalemia occurrences of the 10 patients. Overall, 24 episodes of hyperkalemia were observed in these 10 patients. Of 24 episodes of hyperkalemia, 21 were observed during treatment and 3 were observed after the end of treatment. There were 2 , $5,5,2,4,3$ episodes of hyperkalemia in $0-4,5-8,9-12$, 13-16, 17-20, 21-24 weeks, respectively. Three episodes of hyperkalemia were observed post-treatment at week 1,2 and 3 post-treatment. In summary, hyperkalemia could occur at any time of the observational period from on-treatment to post-treatment.

Of the 10 patients, 2 showed history of hyperkalemia before antiviral treatment. Then, we tested if the history of hyperkalemia before treatment could increase the possibility of hyperkalemia on treatment. A total of $7 \mathrm{ep}-$ isodes of hyperkalemia were observed in the 2 patients with hyperkalemia history, while 17 episodes of hyperkalemia were observed in the rest of 8 patients without hyperkalemia history. Non-parametric test was used to compare hyperkalemia episodes between the patients with and without hyperkalemia history pre-treatment. The median frequencies of hyperkalemia episodes in patients with and without hyperkalemia history pretreatment were 3.5 and 2, respectively. No significant differences between the patients with and without hyperkalemia history pre-treatment $(p=0.264)$.

\section{Discussion and conclusion}

This is the first time to report recurrent hyperkalemia in patients with $\mathrm{CKD}$ and $\mathrm{HCV}$ infection treated with half-dose of sofosbuvir based regimen, although bradycardia, changed intracellular $\mathrm{Ca}^{2+}$ and acute interstitial

Table 1 Demographics of the 10 patients at baseline

\begin{tabular}{|c|c|c|c|c|c|}
\hline Patient & $\begin{array}{l}\text { Gender (F/ } \\
\text { M) }\end{array}$ & $\begin{array}{l}\text { Age } \\
\text { (Years) }\end{array}$ & Underlying Diseases & $\begin{array}{l}\text { \#Dialysis } \\
\text { History }\end{array}$ & Co-medication \\
\hline No. 1 & M & 72 & Diabetes, Hypertension & 3 years & atorvastatin, nifedipine, metoprolol tartrate, caltrate, insulin \\
\hline No. 2 & M & 56 & $\begin{array}{l}\text { Diabetes, Hypertension, } \\
\text { Nephrolithiasis, Coronary heart disease }\end{array}$ & 4 years & nifedipine, carvedilol, novolin 30R \\
\hline No. 3 & $\mathrm{~F}$ & 56 & Diabetes, Hypertension & 4 years & nifedipine, metoprolol tartrate, carvedilol, novolin 30R \\
\hline No. 4 & M & 57 & Nephritis, Hypertension & 4 years & nifedipine, metoprolol tartrate, carvedilol, amlodipine \\
\hline No. 5 & $\mathrm{~F}$ & 54 & Nephritis, Hypertension & 4 years & nifedipine, metoprolol tartrate, carvedilol \\
\hline No. 6 & M & 57 & $\begin{array}{l}\text { Obstructive Nephropathy, } \\
\text { Hydronephrosis, Chronic hepatitis B }\end{array}$ & 1.5 years & None \\
\hline No. 7 & M & 45 & Hydronephrosis & 2 years & metoprolol tartrate, vitamin B12, folic acid \\
\hline No. 8 & M & 43 & Nephritis; Hypertension & 1 years & nifedipine, metoprolol tartrate, carvedilol \\
\hline No. 9 & $\mathrm{~F}$ & 27 & Diabetes, Hypertension & 3 years & $\begin{array}{l}\text { nifedipine, amlodipine, metoprolol tartrate, caltrate, } \\
\text { calcitriol, vitamin B12, folic acid, polysaccharide-iron complex }\end{array}$ \\
\hline No. 10 & M & 25 & $\begin{array}{l}\text { Nephritis, Hypertension } \\
\text { Coronary heart disease, Chronic } \\
\text { Hepatitis B }\end{array}$ & 2 years & nifedipine, metoprolol tartrate, caltrate \\
\hline
\end{tabular}

The antiviral therapy was half dose of sofosbuvir $(200 \mathrm{mg})$ daily or post-dialysis and full dose of daclatasvir $(60 \mathrm{mg})$

\# Dialysis scheme was 5 times per 2 weeks for these patients with stage 5 chronic kidney disease 


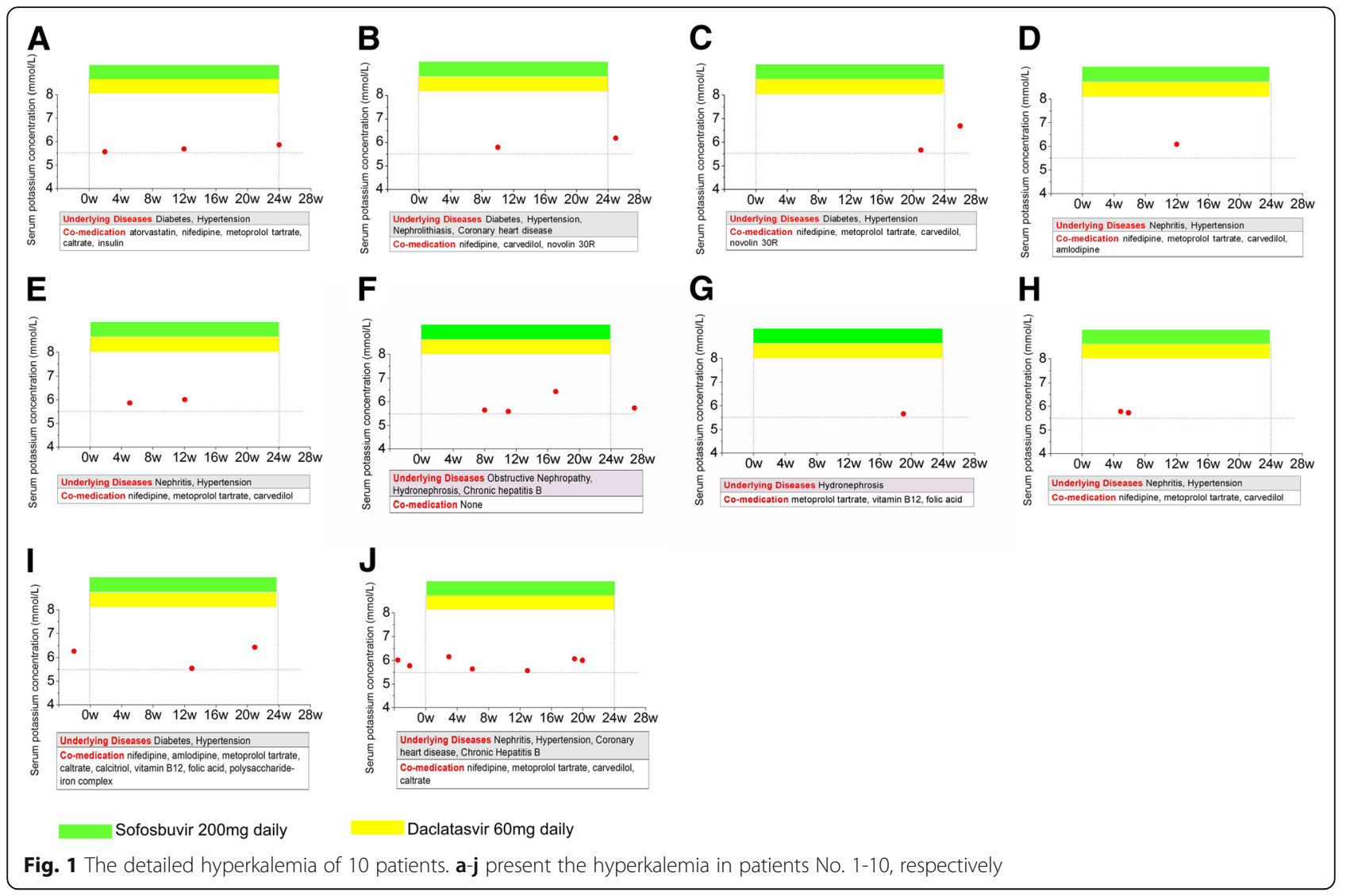

nephritis have been reported potentially associated with sofosbuvir.

The recurrence of hyperkalemia was highly related to the administration of sofosbuvir. Patients with CKD especially in uremia period are at high risk of hyperkalemia, while regular dialysis aims to replace the function of kidney and could maintain the balance of electrolyte. In our case serials, we cannot attribute recurrence of hyperkalemia to insufficiently dialysis, for the dialysis scheme was unchanged before, during and after antiviral treatment. The dialysis therapy was sufficient for these 10 patients; because the serum creatinine levels remained stable and majority $(8 / 10)$ of the patients were not observed hyperkalemia prior to sofosbuvir based treatment. Furthermore, other electrolytes, such as serum sodium or serum calcium disorder was not observed, and acid-base kept in homeostasis, supporting the adequacy of hemodialysis. Moreover, after the end of treatment, 8 out of the 10 patients did not present hyperkalemia, and along with the time extension of drug cessation, no hyperkalemia was observed. The temporal consistency between the DAAs usage and hyperkalemia occurrence highly supported the association between DAAs and hyperkalemia. Based on the HEP Drug Interactions database, no clinically significant interaction was showed between the co-medicines. Collectively, the raising levels of serum potassium were highly suspected in association to the usage of sofosbuvir.

The mechanism of the observed hyperkalemia in patient with CKD and HCV infection treated with sofosbuvir based regimens was unclear. As mentioned above, the recurrence of hyperkalemia could not be attributed to the insufficiency dialysis. Previously, case serials of bradycardia resulted from the co-administration of sofosbuvir and amiodarone were reported [6]. Regan CP et al. and Millard DC et al. demonstrated that coadministration of sofosbuvir and amiodarone produced dysfunction of the sinoatrial node automaticity and atrioventricular node conduction, decrease in cardiomyocyte mechanical activity and intracellular $\mathrm{Ca}^{2+}$ transients $[7,8]$. Acute interstitial nephritis revealed by renal biopsy was reported potentially related to sofosbuvir, we assume that potassium exchange between inside and outside of cells may be an important factor of hyperkalemia $[9,10]$. Actually, although no literature reports the impact on potassium channel by sofosbuvir, high dose of GS-9851 (the parent of sofosbuvir) did inhibit hERGmediated potassium current in HEK293 cells expressing cloned hERG channels [4]. Integrating these evidences together, sofosbuvir do induce the dysfunction of ion channel, and such impact is more prominent in those cases with CKD and co-administered with drugs acting 
on an ion channel. In current report, calcium-channel blockers (CCBs) were administered in 7 patients. The drug-drug interaction could not be neglected in patients with stage 5 CKD co-administered with sofosbuvir and CCBs, since the metabolic pathway of $\mathrm{CCBs}$ have an intersection with that of sofosbuvir.

This is a retrospective report, the occurrence of hyperkalemia may be underestimated especially in patients with stage $5 \mathrm{CKD}$. However, the observation of this report could still provide a clue for clinicians to strengthen the monitoring for serum potassium when treating $\mathrm{HCV}$ infection with sofosbuvir, even its dose is halved, based regimens in patients with CKD.

\section{Abbreviations}

AEs: Adverse effects; CCBs: Calcium-channel blockers; CKD: Chronic kidney disease; DAAs: Direct antiviral agents; ESRD: End-stage renal disease; HCV: Hepatitis C virus

\section{Acknowledgments}

We all express our gratitude to the patients, who kindly gave consent for the cases to be presented in this paper.

\section{Authors' contributions}

TTY, JPW and YLH planned and designed the study and wrote the protocol. YLH, YRZ, TYC and JPW were responsible for the treatment of those patients. $T T Y, Z T, F H Z, J D, J F L$, and $Y Y$ participated in the study monitoring and management. JL, SF, YC, CHH and RZ were biostatisticians and participated in the data analysis and writing of the report. All authors read and approved the final version of the report.

\section{Funding}

This work was supported by the National Natural Science Foundation of China (Grant/Award Number: 81570528, 8177031729) and the grants from the Fundamental Research Funds for the Central Universities.

\section{Availability of data and materials}

The datasets used during the current study are available from the corresponding author on reasonable request.

\section{Ethics approval and consent to participate}

The study protocol was performed to conform with the Declaration of Helsinki and was approved by the Ethics Committee of the First Affiliated Teaching Hospital, SOM, Xi'an Jiaotong University. Written informed consent was obtained from all the patients and/or their relatives.

\section{Consent for publication}

Written informed consent was obtained from the patients and/or relatives for publication of this case report.

\section{Competing interests}

The authors declare that they have no competing interests.

\section{Author details}

${ }^{1}$ Institution of Hepatology, First Affiliated Teaching Hospital, SOM, Xi'an Jiaotong University, Xi'an City, China. ${ }^{2}$ Department of Infectious Diseases, First Affiliated Teaching Hospital, SOM, Xi'an Jiaotong University, No. 277 Yanta Road(w), Xi'an City, Shaanxi Province, China. ${ }^{3}$ Department of Infectious Diseases, Xijing Hospital of the Air Force Medical University, Xi'an City, China. ${ }^{4}$ Department of Internal Medicine, Zhen'An County Hospital, Zhen'An, China. ${ }^{5}$ Department of Haemodialysis, Zhen'An County Hospital, Zhen'An, China.
Received: 19 February 2019 Accepted: 21 May 2019

Published online: 21 June 2019

\section{References}

1. European Association for the Study of the Liver. Electronic address eee, European Association for the Study of the L: EASL recommendations on treatment of hepatitis C 2018. J Hepatol. 2018;69(2):461-511.

2. Bhamidimarri KR, Czul F, Peyton $A$, Levy $C$, Hernandez M, Jeffers $L$, Roth $D$, Schiff E, O'Brien C, Martin P. Safety, efficacy and tolerability of half-dose sofosbuvir plus simeprevir in treatment of hepatitis $C$ in patients with end stage renal disease. J Hepatol. 2015;63(3):763-5.

3. Desnoyer A, Pospai D, Le MP, Gervais A, Heurgue-Berlot A, Laradi A, Harent S, Pinto A, Salmon D, Hillaire S, et al. Pharmacokinetics, safety and efficacy of a full dose sofosbuvir-based regimen given daily in hemodialysis patients with chronic hepatitis C. J Hepatol. 2016:65(1):40-7.

4. Gilead Sciences I. Pharmacology review(s), Sofosbuvir (GS-7977), 204671Orig1s000. 2013. https://www.accessdata.fda.gov/drugsatfda_docs/ nda/2013/204671Orig1 s000PharmR.pdf

5. He YL, Yang SJ, Hu CH, Dong J, Gao H, Yan TT, Liu JF, Yang Y, Ren DF, Zhu $L$, et al. Safety and efficacy of sofosbuvir-based treatment of acute hepatitis $C$ in end-stage renal disease patients undergoing haemodialysis. Aliment Pharmacol Ther. 2018:47(4):526-32

6. Administration USFaD. FDA Drug Safety Communication: FDA warns of serious slowing of the heart rate when antiarrhythmic drug amiodarone is used with hepatitis $C$ treatments containing sofosbuvir Harvoni or Sovaldi in combination with another direct acting antiviral drug. https://www.fda. gov/drugs/drug-safety-and-availability/fda-drug-safety-communication-fdawarns-serious-slowing-heart-rate-when-antiarrhythmic-drug.

7. Millard DC, Strock CJ, Carlson CB, Aoyama N, Juhasz K, Goetze TA, Stoelzle-Feix S, Becker N, Fertig N, January $C T$, et al. Identification of Drug-Drug interactions in vitro: a case study evaluating the effects of Sofosbuvir and amiodarone on hiPSC-derived cardiomyocytes. Toxicol Sci. 2016;154(1):174-82

8. Regan CP, Morissette P, Regan HK, Travis JJ, Gerenser P, Wen JZ, Fitzgerald K, Gruver S, DeGeorge JJ, Sannajust FJ. Assessment of the clinical cardiac drug-drug interaction associated with the combination of hepatitis $C$ virus nucleotide inhibitors and amiodarone in Guinea pigs and rhesus monkeys. Hepatology. 2016;64(5):1430-41.

9. Wanchoo R, Thakkar J, Schwartz D, Jhaveri KD. Harvoni (Ledipasvir with Sofosbuvir)-induced renal injury. Am J Gastroenterol. 2016;111(1):148-9.

10. Ashraf T, Majoni W. Acute interstitial nephritis associated with Sofosbuvir and Daclatasvir. ACG Case Rep J. 2017:4:e84.

\section{Publisher's Note}

Springer Nature remains neutral with regard to jurisdictional claims in published maps and institutional affiliations.
Ready to submit your research? Choose BMC and benefit from:
- fast, convenient online submission
- thorough peer review by experienced researchers in your field
- rapid publication on acceptance
- support for research data, including large and complex data types
- gold Open Access which fosters wider collaboration and increased citations
- maximum visibility for your research: over $100 \mathrm{M}$ website views per year
At BMC, research is always in progress.
Learn more biomedcentral.com/submission 